A. Pramesh Rao, G. Swarup and Gopal-Krishna, eds.

\title{
HI Absorption in Radio Galaxies
}

\author{
R. Morganti, T.A. Oosterloo \\ Netherlands Foundation for Research in Astronomy, Postbus 2, 7990 \\ AA, Dwingeloo, The Netherlands \\ G. van Moorsel \\ National Radio Astronomy Observatory, Socorro, NM 87801, USA \\ C.N. Tadhunter \\ Dept. Physics, University of Sheffield, Sheffield S3 $7 R H, U K$ \\ N. Killeen \\ Australia Telescope National Facility, CSIRO, P.O. Box 76, 2121 \\ Epping NSW, Australia
}

\begin{abstract}
Twenty-two powerful radio galaxies have been searched for $\mathrm{HI}$ absorption. We find the highest probability of detecting $\mathrm{HI}$ in absorption among narrow-line compact (or small) galaxies or galaxies with indication of richer interstellar medium (i.e. with ongoing or recent starformation). We discuss the difficulty in the interpretation of the origin of the HI absorption due to the uncertainty in the systemic velocity of the galaxies.
\end{abstract}

A number of interesting features and phenomena are taking place in the central regions of Active Galactic Nuclei (AGN). Among others, and relevant for this work, are: i) the presence of nuclear tori (on the pc-scale) and larger scale disk/tori (extending up to kpc scale and beyond), ii) the possible presence of in-falling gas that has been often suggested as responsible for "feeding" of the central engine, iii) the presence of gas disturbed by the interaction with the radio plasma and finally, iv) the presence of larger-scale tails of gas (that in particular conditions can be seen projected against the nuclear regions) possibly the result of merger or interaction with an other object.

The study of all these phenomena requires kinematical information about the gas and therefore one of the few ways to obtain this is by studying the HI absorption against the nuclei of radio galaxies. The advantage of this technique is that observations of the $\mathrm{HI}$ absorption against radio continuum sources allow to detect relatively small quantities of $\mathrm{HI}$ even in high redshift objects.

\section{A Well-Studied Sample}

Many of the cases of HI absorption detected so far in radio galaxies have been interpreted as evidence for obscuring tori. Thus it can be interesting to investigate 
whether the presence of absorption correlates with other orientation-dependent indicators (like, e.g., the width of the optical lines). In order to do this, we have been looking for $\mathrm{HI}$ absorption in a sample of radio galaxies for which a wealth of radio, optical and $\mathrm{X}$-ray information is available.

The observed radio galaxies have been selected from the 2-Jy sample of radio sources: a summary of the data available on this sample can be found in Tadhunter et al. (1998) and Morganti et al. (1999) and references therein. This sample includes strong $\left(\mathrm{S}_{2.7 \mathrm{GHz}}>2 \mathrm{Jy}\right)$ radio sources with declination $\delta<10^{\circ}$. So far we have look for HI absorption in 22 radio galaxies. The observed objects have been selected to have a strong enough radio core and $z \lesssim 0.2$, i.e. the highest redshift reachable by most currently available $21-\mathrm{cm}$ radio receivers, although the sample is complete up to $z=0.7$. Due to the southern declination of most objects in the sample, only an handful of higher redshift objects could be observed with the UHF system in use at the WSRT. The role of GMRT in extending this study at higher redshift will be essential. The observations so far were done mainly with the Australia Telescope Compact Array (ATCA) and with the Very Large Array (VLA) (depending on the declination of the objects).

\section{Occurrence and origin of the $\mathrm{HI}$ absorption}

Of the 22 radio galaxies for which the data have been analysed so far, we find five galaxies with clear $\mathrm{HI}$ absorption (with optical depths ranging between 2 and $10 \%$ ): PKS 1318-43 (NGC 5090), PKS 1549-79, PKS 1717-00 (3C353), PKS 1814-63 and PKS 2314+03 (3C459). For the undetected galaxies, a $3 \sigma$ limit to the optical depth of at most few percent has been obtained in most cases. Here are more details about the occurrence of the HI absorption.

- Of the 11 Fanaroff-Riley (FR) type-I observed, we detected only one (PKS 1318-43). A few more can be included if the data available in literature are considered (e.g. Hydra A, NGC 4261 etc.).

- So far, in our sample no HI absorption has been detected in broad line radio galaxies (BLRG) (see also below).

- The three compact (or small) radio galaxies observed by us were all detected: PKS 1814-63, PKS 1549-79, 3C459. The HI absorption profiles of the first two are shown in Fig.1. Two more compact sources in the sample were observed with the WSRT (R. Vermeulen private communication). Only one of the two has been detected (PKS 0023-26). However, it is worth mentioning that the undetected source (PKS 2135-20) is a broad line radio galaxy, consistent with the trend for BLRG described above.

- Only two radio galaxies (3C459 and PKS 1549-79) in the whole 2-Jy sample show evidence of a star-burst or young stellar population component in their optical spectra (in addition to the old stellar population and the nonthermal power law component). These galaxies, seems to have a richer interstellar medium (ISM), they are also bright in FIR emission, compared to the other powerful radio galaxies in the sample and $H I$ absorption has been detected in both of them.

The low detection rate of HI absorption in FR type-I galaxies is somewhat surprising given that, as shown from recent HST images, gas and dust, in particular circumnuclear dust-lanes, are commonly present in low-luminosity radio 
PKS 1549-79

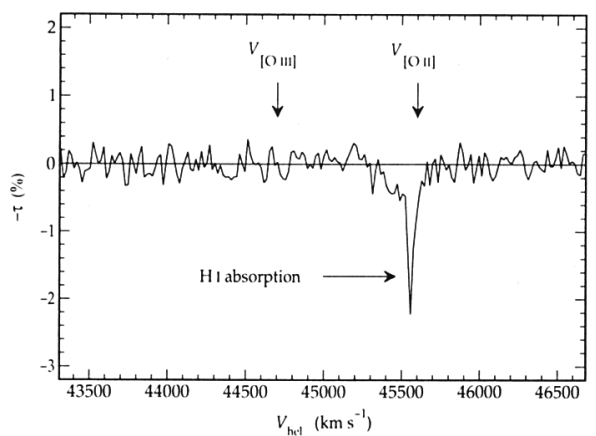

PKS 1814-637

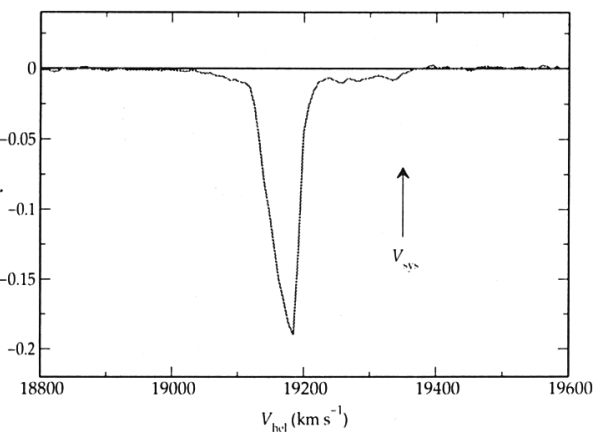

Figure 1. Left: HI absorption spectra (from the ATCA) observed in the source PKS 1549-79. Two different velocities have been derived from the [OIII] and [OII] emission lines. See text for details. Right: HI absorption spectra (from the ATCA) observed in the source PKS 181463. The new systemic velocity is indicated.

galaxies (see e.g. Chiaberge et al. 1999). However, also from optical studies it is clear that the nuclei of FR type-I galaxies appear basically not obscured. Indeed, for this type of radio galaxies the presence of thick disks surrounding the central black hole is not really required by unified schemes (Urry \& Padovani 1995). Thus, our result is consistent with the work of Chiaberge et al. (1999) arguing that the "standard" pc scale, geometrically thick torus is not present in low-luminosity radio galaxies. A possibility is that the circumnuclear disks in these objects are typically thin $(\lesssim 20$ pc) as found in the case of NGC 4261 (Jaffe \& McNamara 1994).

In the case of FR type-II radio galaxies, the presence of a thick disk is a vital ingredient in the unified schemes hypothesis. Therefore, HI absorption is expected in narrow line galaxies. It appears that for powerful radio galaxies the highest probability of detecting $\mathrm{HI}$ in absorption is among narrow-line compact (or small) galaxies (as already pointed out by Conway 1996) or galaxies with a richer interstellar medium (i.e. with ongoing or recent star-formation). Unfortunately, because of the selection criteria, our sample is biased against powerful, extended, narrow line radiogalaxies. This bias prevent us to establish whether the $\mathrm{HI}$ absorption is common also in the extended narrow line radio galaxies or only in the compact ones.

So far, HI absorption seems absent in BLRG. If the HI absorption is due to an obscuring torus or disk, this result is what naively is expected according to the unified schemes. Broad line radio galaxies are in fact supposed to be galaxies seen pole-on and therefore obscuration from the torus should not occur.

Is the absorbing material really situated in a disk/torus? From the average profile we cannot say for sure but the presence of the absorption in narrow-line galaxies and the absence in broad line galaxies seems to be consistent with this possibility. On the other hand the presence of absorption in the case of richer ISM could make the story more complicated. 
In order to really answer this question, imaging of the HI absorption on the $V L B I$ scale together with good values for the systemic velocities are needed.

\section{The Problem of the Systemic Velocity}

It was already pointed out by Mirabel (1989) how the systemic velocities derived from emission lines can be both uncertain and biased by motions connected with the gas producing these emission lines.

We have found a very good example of this uncertainty in one of the objects showing HI absorption: PKS 1549-79. In this galaxy the available redshift derived from [OIII] emission lines (that is commonly used for the majority of radio galaxies) would suggest the $\mathrm{HI}$ being strongly redshifted (see Fig.1). A more detailed analysis of the spectrum has shown that the [OII] lines give a velocity about $800 \mathrm{~km} / \mathrm{s}$ higher compared to what derived from the [OIII] lines and consistent with the HI absorption (see Fig. 1). What should we consider as systemic velocity of the galaxy? The [OIII] lines appear to be very broad, one of the broadest observed in radio galaxies with a FWHM of $\sim 1300 \mathrm{~km} / \mathrm{s}$. Thus, we belive that much of the [OIII] is emitted by an inner narrow line region, which is undergoing outflow from the nucleus, whereas the [OII] are likely to be closer to the real systemic velocity of this galaxy.

Also in the case of the other compact object PKS 1814-637 a more accurate redshift has been derived. The HI absorption appears now to be mainly blueshifted (unlike from the value derived using the old systemic velocity) as shown in Fig. 1. With this new piece of information the HI absorption, and in particular the broad component visible at about $1 \%$ level optical depth, could be due to some gas outflow connected perhaps to interaction between the radio plasma and the ISM. HI absorption due to gas outflow has been clearly seen already in few objects as the Seyfert galaxy IC 5063 (Oosterloo et al. 1999), the superluminal source 3C216 (Pihlström et al.1999) and 3C326 (Conway et al. in prep).

So far, we have not found objects with clear redshifted HI absorption.

\section{References}

Chiaberge M., Capetti A., Celotti A., 1999, A\&A 349, 77

Conway J.E. 1996, in The second Workshop on GPS and CSS Radio Sources, Eds. Snellen, Schilizzi et al. M.N. Publ JIVE, Leiden p.198

Jaffe W. \& McNamara B. 1994, ApJ, 434, 110

Mirabel I.F. 1989, ApJ 340, L13

Morganti R., Oosterloo T.A., Tadhunter C.N., Aiudi R., Jones P., Villar-Martin 1999, A\&A Suppl. 140, 355

Oosterloo T.A., Morganti R., Tzioumis A., Reynolds J., King E., McCulloch P., Tsvetanov Z. 1999, AJ in press

Pihlström Y.M., Vermeulen R.C., Taylor G.B., Conway J.E. 1999, ApJ 525, L13 Tadhunter C.N., Morganti R., Robinson A., Dickson R., Villar-Martin M. \& Fosbury R.A.E., 1998, MNRAS 298, 1035 